\title{
Zeros of Hecke $L$-functions associated with cusp forms
}

\author{
by \\ Wenzhi Luo (Berkeley, Calif.)
}

1. Introduction. Let $f(z)=\sum_{n=1}^{\infty} a_{n} e(n z)$ be a holomorphic cusp form of even integral weight $k>0$ with respect to the modular group $\Gamma=$ $\mathrm{SL}(2, \mathbb{Z})$, and define (for $\Re s>(k+1) / 2$ )

$$
L_{f}(s)=\sum_{n=1}^{\infty} a_{n} n^{-s},
$$

the associated Hecke $L$-function. We also assume that $f(z)$ is a Hecke eigenform [11] with $a_{1}=1$. Recall that we have the bound for the coefficients

$$
\left|a_{n}\right| \leq d(n) n^{(k-1) / 2}
$$

by Deligne's proof of the Ramanujan-Petersson conjecture [2], [3], and the bound for the square mean [9], [18],

$$
\sum_{n \leq N}\left|a_{n}\right|^{2} \ll N^{k}
$$

It is well known [10] that $L_{f}(s)$ admits analytic continuation to $\mathbb{C}$ as an entire function and satisfies the functional equation

$$
(2 \pi)^{-s} \Gamma(s) L_{f}(s)=(-1)^{k / 2}(2 \pi)^{-(k-s)} \Gamma(k-s) L_{f}(k-s) .
$$

Moreover, $L_{f}(s)$ has Euler product representation $(\Re s>(k+1) / 2)$

$$
L_{f}(s)=\prod_{p}\left(1-a_{p} p^{-s}+p^{k-1} p^{-2 s}\right)^{-1} .
$$

The non-trivial zeros of $L_{f}(s)$ lie within the strip $(k-1) / 2<\Re s<(k+1) / 2$, symmetrically to the real axis and the critical line $\sigma=k / 2$. The Riemann Hypothesis for $L_{f}(s)$ asserts that all the non-trivial zeros of $L_{f}(s)$ lie on the critical line $\Re s=k / 2$. Hafner [13], generalizing Selberg's remarkable work [19] on $\zeta(s)$, has shown that a positive proportion of all non-trivial zeros are on the critical line.

The research of the author was supported by NSF Grant DMS 9304580, while he was a member at the Institute for Advanced Study in Princeton during 1993-1994. 
In this work, we establish the analogue of Selberg's density theorem [20] for $L_{f}(s)$. Define, for $\sigma \geq k / 2$ and $T \geq 1$,

$$
N_{f}(\sigma, T)=\left|\left\{\beta+i \gamma: L_{f}(\beta+i \gamma)=0, \beta \geq \sigma, 0<\gamma \leq T\right\}\right| .
$$

It was proved by Lekkerkerker [15] that

$$
N_{f}\left(\frac{k-1}{2}, T\right) \sim \frac{1}{\pi} T \log T .
$$

We will show that

TheOREM 1.1. For some $a>0$ we have

$$
N_{f}(\sigma, T) \ll T^{1-a(\sigma-k / 2)} \log T,
$$

uniformly for $k / 2 \leq \sigma \leq(k+1) / 2$.

Our proof shows that one may take $a=1 / 72$. However, we make no effort to obtain an optimal $a$ by our method.

Application of standard techniques of analytic number theory easily yields results of the type

$$
N_{f}(\sigma, T) \ll T^{c(\sigma)}(\log T)^{A},
$$

where $c(\sigma)<1$ for $\sigma>k / 2$ and some $A>0$. The significance of Theorem 1.1 lies in that $A$ can be taken to be 1. Selberg used the analogue of Theorem 1.1 for $\zeta(s)$ to prove his famous result on the moments of $\arg \zeta(1 / 2+i t)$. In view of recent work of Bombieri and Hejhal [1], there is a similar application to $\arg L_{f}(k / 2+i t)$, which is the main motivation of the present paper.

COROllary 1.2. The functions

$$
\frac{\log \left|L_{f}(k / 2+i t)\right|}{\sqrt{\pi \log \log t}}, \quad \frac{\arg L_{f}(k / 2+i t)}{\sqrt{\pi \log \log t}}
$$

become distributed, in the limit of large $t$, like independent random variables, each having Gaussian density $\exp \left(-\pi u^{2}\right) d u$.

To prove Theorem 1.1 by Selberg's method, one considers not $L_{f}(s)$ itself, but $L_{f}(s) M_{X}(s)$, where the mollifier $M_{X}(s)$ is a Dirichlet polynomial of length $X=T^{\theta}, \quad 0<\theta<1 / 2$, and is chosen such that $L_{f}(s) M_{X}(s)$ is very close to 1 in the region $\sigma>k / 2,0<t \leq T$, or more precisely such that the mean value

$$
\frac{1}{T} \int_{T}^{2 T}\left|L_{f}(\sigma+i t) M_{X}(\sigma+i t)-1\right|^{2} d t
$$

is very small, i.e.

$$
\int_{T}^{2 T}\left|L_{f}(\sigma+i t) M_{X}(\sigma+i t)-1\right|^{2} d t \ll T^{1-a(\sigma-k / 2)},
$$


uniformly for $k / 2 \leq \sigma \leq(k+1) / 2$. It is then possible to deduce, by a standard argument (see $\S 3$ ), that the zeros of $L_{f}(s) M_{X}(s)$ and a fortiori of $L_{f}(s)$ in the region considered are comparatively few. The required mean value estimate is obtained as follows. If we prove

$$
\int_{T}^{2 T}\left|L_{f}(k / 2+i t) M_{X}(k / 2+i t)-1\right|^{2} d t \ll T,
$$

and

$$
\int_{T}^{2 T}\left|L_{f}(k / 2+1+i t) M_{X}(k / 2+1+i t)-1\right|^{2} d t \ll T^{1-a},
$$

then by a convexity theorem (see $\S 3$ ) we have

$$
\int_{T}^{2 T}\left|L_{f}(\sigma+i t) M_{X}(\sigma+i t)-1\right|^{2} d t \ll T^{1-a(\sigma-k / 2)},
$$

uniformly for $k / 2 \leq \sigma \leq k / 2+1$, which is all we need. The second inequality is easy to prove since $L_{f}(s)$ is an absolutely convergent Dirichlet series for $\Re s>(k+1) / 2$ and $M_{X}(s)$ is an approximate inverse to $L_{f}(s)$ such that $L_{f}(s) M_{X}(s)-1$ is given by a Dirichlet series of the type $\sum_{n \geq y} b_{n} n^{-s}$, with $y$ large. The first inequality represents the main difficulty, since it is not obtainable by a routine extension of Selberg's work in the $\zeta(s)$ case. We will replace $L_{f}(s)$ by a Dirichlet polynomial of length $\sim T$ using the approximate functional equation of $L_{f}(s)$ in the form obtained by A. Good [7]. The resulting expression then becomes

$$
\int_{T}^{2 T}\left|P_{T X}(\sigma+i t)\right|^{2} d t
$$

where $P_{T X}$ is a Dirichlet polynomial of length $T X$. However, in general no method succeeds in handling the above mean value once $P(s)$ has length $\gg T$. Therefore we have to make careful use of the special feature of $M_{X}(s)$. In fact, the argument similar to Selberg [19] and Hafner [13], with some modification, is suitable here. For some technical reason we will prove the first inequality for $\sigma=k / 2+1 / \log T$ rather than $k / 2$ and then apply a convexity theorem to obtain Theorem 1.1.

We remark here that in our proof of Theorem 1.1 Deligne's bound for the Fourier coefficients $a_{n}$ is used but not crucial here. A weaker bound like $a_{n} \ll n^{(k-1) / 2+1 / 4+\varepsilon}$ which follows from Weil's bound for the Kloosterman sums and the bound for the square mean mentioned before would suffice. Thus, our method should be applicable when $f(z)$ is a Maass form, though the Ramanujan-Petersson conjecture remains unproved in this case. 
We would like to mention that D. Farmer [6], using Hafner's method and the spectral theory, establishes an asymptotic formula for the mean square of $L_{f}(s)$ weighted by a general mollifier of Levinson's type. He mentions that this mean value theorem can be combined with Jutila's method [14] to give a density result, but he does not give any details.

The author would like to express his gratitude to Professor E. Bombieri, for suggesting this problem to him, and for constant encouragement throughout this work.

The author is grateful for Professor A. Selberg's helpful remarks, especially for suggesting to the author a much simpler choice of the mollifier and the use of the convexity theorem, leading to substantial simplification of an earlier version of this work.

The author would also like to thank Professor H. Iwaniec and Professor P. Sarnak for some helpful comments.

2. Main lemma. Let $\psi_{U}(t)$ be a non-negative smooth function such that

and

$$
\psi_{U}(t)= \begin{cases}0 & \text { if } t \leq 1-1 / U \text { or } t \geq 2+1 / U \\ 1 & \text { if } 1+1 / U \leq t \leq 2-1 / U\end{cases}
$$

$$
\psi_{U}^{(p)}(t) \ll U^{p}, \quad p \geq 0,
$$

where $U$ is a positive parameter and in our discussion it will be chosen as $O(1)$ later. The object of this section is to prove the following lemma, which is the analogue of Lemma 6 in [19].

Lemma 2.1. If $k / 2<\sigma \leq k / 2+1 / 40, \varepsilon>0$, and $\mu, \nu$ are positive coprime integers $\leq T$, then

$$
\begin{aligned}
\int_{-\infty}^{\infty} \psi_{U}\left(\frac{t}{T}\right) & \left|L_{f}(\sigma+i t)\right|^{2}\left(\frac{\mu}{\nu}\right)^{i t} d t \\
= & \frac{1}{(\mu \nu)^{\sigma}} D_{\mu \nu}(2 \sigma) \int_{-\infty}^{\infty} \psi_{U}\left(\frac{t}{T}\right) d t \\
& +\frac{1}{(\mu \nu)^{k-\sigma}} D_{\mu \nu}(2 k-2 \sigma) \int_{-\infty}^{\infty} \psi_{U}\left(\frac{t}{T}\right)\left(\frac{t}{2 \pi}\right)^{2 k-4 \sigma} d t \\
& +O\left(\frac{(\mu \nu)^{3} U^{4} T^{4 / 5}}{2 \sigma-k}\right),
\end{aligned}
$$

where

$$
D_{\mu \nu}(s)=\sum_{l=1}^{\infty} \frac{a_{\mu l} a_{\nu l}}{l^{s}} .
$$


Proof. The proof is very similar to the treatment in [8] and [13], and so we give only a sketch. We have (denote $\sigma+i t$ by $s$ )

$$
\begin{aligned}
& \int_{-\infty}^{\infty} \psi_{U}\left(\frac{t}{T}\right)\left|L_{f}(\sigma+i t)\right|^{2}\left(\frac{\mu}{\nu}\right)^{i t} d t \\
& =\int_{-\infty}^{\infty} \psi_{U}\left(\frac{t}{T}\right) L_{f}(\sigma+i t) L_{f}(\sigma-i t)\left(\frac{\mu}{\nu}\right)^{i t} d t \\
& =\int_{-\infty}^{\infty}\left(\sum_{n=1}^{\infty} a_{n} n^{-\sigma}(n \nu)^{-i t} \phi\left(\frac{2 \pi n}{t} \sqrt{\frac{\nu}{\mu}}\right)\right. \\
& \left.\quad+(2 \pi)^{2 s-k} \frac{\Gamma(k-s)}{\Gamma(s)} \sum_{n=1}^{\infty} a_{n} n^{\sigma-k}\left(\frac{n}{\nu}\right)^{i t} \phi^{*}\left(\frac{2 \pi n}{t} \sqrt{\frac{\mu}{\nu}}\right)\right) \\
& \quad \times\left(\sum_{m=1}^{\infty} a_{m} m^{-\sigma}(m \mu)^{i t} \phi\left(\frac{2 \pi m}{t} \sqrt{\frac{\mu}{\nu}}\right)\right. \\
& \left.\quad+(2 \pi)^{2 \bar{s}-k} \frac{\Gamma(k-\bar{s})}{\Gamma(\bar{s})} \sum_{m=1}^{\infty} a_{m} m^{\sigma-k}\left(\frac{m}{\mu}\right)^{-i t} \phi^{*}\left(\frac{2 \pi m}{t} \sqrt{\frac{\nu}{\mu}}\right)\right) \psi_{U}\left(\frac{t}{T}\right) d t \\
& \quad+O\left(\left(\sqrt{\frac{\mu}{\nu}}+\sqrt{\frac{\nu}{\mu}}\right) \log ^{2} T\right) .
\end{aligned}
$$

Here we use the approximate functional equation for $L_{f}(\sigma \pm i t)$ (see [7], Satz), and $\phi(\xi), \phi^{*}(\xi)$ are suitable smooth functions satisfying $\phi^{*}(\xi)=1-\phi(1 / \xi)$, and $\phi(\xi)=1,|\xi| \leq 2 / 3 ; \phi(\xi)=0,|\xi| \geq 3 / 2$.

Multiplying out the expression in the above integrand and using the same argument and notation as in [8], §2 (see also [13], §3), we see that the above expression equals

$$
\begin{aligned}
\sum_{n, m} \frac{a_{n} a_{m}}{(n m)^{\sigma}} & \int_{-\infty}^{\infty} \psi_{U}\left(\frac{t}{T}\right)\left(\frac{m \mu}{n \nu}\right)^{i t} \Phi\left(\frac{2 \pi n}{t} \sqrt{\frac{\nu}{\mu}}, \frac{2 \pi m}{t} \sqrt{\frac{\mu}{\nu}}\right) d t \\
& +\sum_{n, m} \frac{a_{n} a_{m}}{(n m)^{k-\sigma}}(2 \pi)^{-2 k+4 \sigma} \\
& \times \int_{-\infty}^{\infty} t^{2 k-4 \sigma} \psi_{U}\left(\frac{t}{T}\right)\left(\frac{n \mu}{m \nu}\right)^{i t} \Phi^{*}\left(\frac{2 \pi n}{t} \sqrt{\frac{\mu}{\nu}}, \frac{2 \pi m}{t} \sqrt{\frac{\nu}{\mu}}\right) d t \\
& +O\left(\frac{\log ^{2} T}{2 \sigma-k}\left(\sqrt{\frac{\nu}{\mu}}+\sqrt{\frac{\mu}{\nu}}\right)\right)=S_{1}+S_{2}+S_{3},
\end{aligned}
$$

say, where $\Phi, \Phi^{*}$ are certain smooth functions with compact supports, and $\Phi(\varrho, \varrho)=\phi(\varrho), \Phi^{*}(\varrho, \varrho)=\phi^{*}(\varrho)$. 
For $S_{1}$, the terms with $m \mu=n \nu$ give

$$
\sum_{l=1}^{\infty} \frac{a_{\mu l} a_{\nu l}}{\left(\mu \nu l^{2}\right)^{\sigma}} \int_{-\infty}^{\infty} \psi_{U}\left(\frac{t}{T}\right) \phi\left(\frac{2 \pi \sqrt{\mu \nu} l}{t}\right) d t .
$$

For the terms with $m \mu \neq n \nu$, we let $m \mu-n \nu=l$. Without loss of generality we may assume $l>0$. Then the non-diagonal terms with $l>0$ give

(2) $\quad \sum_{l>0} \sum_{n=1}^{\infty} \frac{a_{n} a_{(n \nu+l) / \mu}}{\left(n \frac{n \nu+l}{\mu}\right)^{\sigma}}$

$$
\begin{aligned}
& \times \int_{-\infty}^{\infty} \psi_{U}\left(\frac{t}{T}\right)\left(\frac{n \nu+l}{n \nu}\right)^{i t} \Phi\left(\frac{2 \pi n}{t} \sqrt{\frac{\nu}{\mu}}, \frac{2 \pi(n \nu+l)}{t \sqrt{\mu \nu}}\right) d t \\
= & (\mu \nu)^{\sigma} \sum_{0<l \leq \sqrt{\mu \nu} U T^{\varepsilon}} \sum_{n=1}^{\infty} \frac{a_{n} a_{(n \nu+l) / \mu}}{(n \nu+l / 2)^{2 \sigma}} \\
& \times \int_{-\infty}^{\infty} \psi_{U}\left(\frac{t}{T}\right) \phi\left(\frac{2 \pi(n \nu+l / 2)}{t \sqrt{\mu \nu}}\right) e^{i t \frac{l}{n \nu+l / 2}} d t \\
& +O\left(\frac{(\mu \nu)^{3} U^{4} \log ^{2} T}{2 \sigma-k}\right) .
\end{aligned}
$$

For $S_{2}$, the terms $n \mu=m \nu$ give

$$
(2 \pi)^{-2 k+4 \sigma} \sum_{l=1}^{\infty} \frac{a_{\mu l} a_{\nu l}}{\left(\mu \nu l^{2}\right)^{k-\sigma}} \int_{0}^{\infty} \psi_{U}\left(\frac{t}{T}\right) \phi^{*}\left(\frac{2 \pi \sqrt{\mu \nu} l}{t}\right) t^{2 k-4 \sigma} d t .
$$

For the terms with $n \mu \neq m \nu$, we let $m \nu-n \mu=l$, and the non-diagonal terms with $l>0$ give

$$
\begin{aligned}
& (4))^{k-\sigma}(2 \pi)^{-2 k+4 \sigma} \sum_{0<l \leq \sqrt{\mu \nu} U T^{\varepsilon}} \sum_{n=1}^{\infty} \frac{a_{n} a_{(n \mu+l) / \nu}}{(n \mu+l / 2)^{2 k-2 \sigma}} \\
& \times \int_{-\infty}^{\infty} \psi_{U}\left(\frac{t}{T}\right) t^{2 k-4 \sigma} \phi^{*}\left(\frac{2 \pi(n \mu+l / 2)}{t \sqrt{\mu \nu}}\right) e^{-i t \frac{l}{n \nu+l / 2}} d t+O\left(\frac{(\mu \nu)^{3} U^{4} \log ^{2} T}{2 \sigma-k}\right) .
\end{aligned}
$$

Let

$$
G(s)=\int_{0}^{\infty} \phi(x) x^{s-1} d x, \quad G^{*}(s)=\int_{0}^{\infty} \phi^{*}(x) x^{s-1} d x .
$$

Then, by Mellin inversion,

$$
\phi(x)=\frac{1}{2 \pi i} \int_{(2)} G(s) x^{-s} d s, \quad \phi^{*}(x)=\frac{1}{2 \pi i} \int_{(2)} G^{*}(s) x^{-s} d s .
$$


Note that $G(s)$ and $G^{*}(s)$ are analytic except for a simple pole at $s=0$ with residue 1 , and $D_{\mu \nu}(s)$ has only a simple pole at $s=k$ for $\Re s \geq k-1 / 2$,

$$
\begin{gathered}
D_{\mu \nu}(s)=P(\mu, s) P(\nu, s) D(s) \quad \text { with } D(s)=\sum_{l=1}^{\infty} a_{l}^{2} l^{-s} ; \\
P(a, s)=\prod_{p^{r} \| a}\left(\sum_{j=0}^{\infty} a_{p^{r+j}} a_{p^{j}} p^{-j s}\right)\left(\sum_{j=0}^{\infty} a_{p^{j}}^{2} p^{-j s}\right)^{-1} ; \\
D(s) \ll t^{1+\varepsilon}, \quad \Re s \geq k-1 / 2 ; \\
P(a, s) \ll a^{(k-1) / 2+\varepsilon}, \quad \Re s \geq k-1 / 2 ; \\
|G(s)|+\left|G^{*}(s)\right| \ll l \frac{1}{|s(s+1) \ldots(s+l)|} .
\end{gathered}
$$

Thus (1) and (3) equal respectively

$$
\begin{gathered}
\frac{1}{(\mu \nu)^{\sigma}} \cdot \frac{1}{2 \pi i} \int_{(2)}\left(\frac{1}{2 \pi \sqrt{\mu \nu}}\right)^{s} G(s) D_{\mu \nu}(2 \sigma+s) \int_{-\infty}^{\infty} \psi_{U}\left(\frac{t}{T}\right) t^{s} d t \\
\frac{1}{(\mu \nu)^{k-\sigma}} \cdot \frac{1}{2 \pi i} \int_{(2)}\left(\frac{1}{2 \pi \sqrt{\mu \nu}}\right)^{s} G^{*}(s) D_{\mu \nu}(2(k-\sigma)+s) \\
\times \int_{-\infty}^{\infty} \psi_{U}\left(\frac{t}{T}\right) t^{s}\left(\frac{t}{2 \pi}\right)^{2 k-4 \sigma} d t
\end{gathered}
$$

Next we shift the lines of integration in (5), (6) to $\Re s=-1 / 2$ and $\Re s=$ $-1 / 2-2 k+4 \sigma$, respectively. The integrands have poles at $k-2 \sigma, 0$ and $2 \sigma-k, 0$, respectively. The residues at $k-2 \sigma$ and $2 \sigma-k$ cancel out and the residues at 0 give the main terms.

By the estimate given above, we deduce that

$$
\begin{aligned}
& (5)+(6)=\frac{1}{(\mu \nu)^{\sigma}} D_{\mu \nu}(2 \sigma) \int_{-\infty}^{\infty} \psi_{U}\left(\frac{t}{T}\right) d t \\
& +\frac{1}{(\mu \nu)^{k-\sigma}} D_{\mu \nu}(2 k-2 \sigma) \int_{-\infty}^{\infty} \psi_{U}\left(\frac{t}{T}\right)\left(\frac{t}{2 \pi}\right)^{2 k-2 \sigma} d t+O\left(T^{1 / 2+\varepsilon}\right) .
\end{aligned}
$$

Define

$$
H_{l}(s)=\int_{0}^{\infty} \phi(\xi) e^{2 \pi i(l / \sqrt{\mu \nu}) \xi^{-1}} \xi^{s-1} d \xi .
$$

This is an entire function and by the Mellin inversion formula,

$$
\phi(\xi) e^{2 \pi i(l / \sqrt{\mu \nu}) \xi^{-1}}=\frac{1}{2 \pi i} \int_{(2)} H_{l}(s) \xi^{-s} d s .
$$


Thus the sum in (2) becomes

$$
\begin{aligned}
& (\mu \nu)^{\sigma} \sum_{0<|l| \leq \sqrt{\mu \nu} U T^{\varepsilon}} \sum_{n=1}^{\infty} \frac{a_{n} a_{(n \nu+l) / \mu}}{(n \nu+l / 2)^{2 \sigma}} \\
& \quad \times \int_{-\infty}^{\infty} \psi_{U}\left(\frac{t}{T}\right) \phi\left(\frac{2 \pi(n \nu+l / 2)}{t \sqrt{\mu \nu}}\right) e^{i t l /(n \nu+l / 2)} d t \\
& =(\mu \nu)^{\sigma} \sum_{0<l \leq \sqrt{\mu \nu} U T^{\varepsilon}} \frac{1}{2 \pi i} \int_{(2)}\left(\frac{\sqrt{\mu \nu}}{2 \pi}\right)^{s} H_{l}(s) D_{\mu \nu}(s+2 \sigma, l) \int_{-\infty}^{\infty} \psi_{U}\left(\frac{t}{T}\right) t^{s} d t .
\end{aligned}
$$

Here

$$
D_{\mu \nu}(s, l)=\sum_{n=1}^{\infty} \frac{a_{n} a_{(n \nu+l) / \mu}}{(n \nu+l / 2)^{s}} .
$$

We move the line of integration to $\Re s=-1 / 5$. We have, on $\Re s=-1 / 5$,

$$
\begin{aligned}
\int_{-\infty}^{\infty} \psi_{U}\left(\frac{t}{T}\right) t^{s} d t & \ll \frac{1}{(|s|+1)^{4}} U^{3} T^{4 / 5}, \\
H_{l}(s) & \ll \frac{\sqrt{\mu \nu}}{l}|s|,
\end{aligned}
$$

and we also have Hafner's result [12]

$$
D_{\mu \nu}(2 \sigma+s, l) \ll \frac{l|s|^{1+\varepsilon}}{(\mu \nu)^{k / 2-7 / 4}} .
$$

Thus the above expression is majorized by

$$
\frac{(\mu \nu)^{\sigma}}{(\mu \nu)^{k / 2-7 / 4}} \mu \nu U^{4} T^{4 / 5} \ll(\mu \nu)^{3} U^{4} T^{4 / 5} .
$$

Similarly, we obtain the same bound for the sum occurring in (4). Thus the proof is complete.

In Section 4 we will give an alternative, and more elementary, treatment of $D_{\mu \nu}(s, l)$ giving a slightly weaker analytic continuation result, which still suffices for the proof.

3. Proof of Theorem. Let

$$
L_{f}^{-1}(s)=\sum_{n=1}^{\infty} \frac{\mu_{f}(n)}{n^{s}}, \quad \Re s>\frac{k+1}{2} .
$$

Thus

$$
\mu_{f}\left(p^{r}\right)= \begin{cases}1 & \text { if } r=0 \\ -a_{p} & \text { if } r=1 \\ p^{k-1} & \text { if } r=2 \\ 0 & \text { if } r \geq 3\end{cases}
$$


Set $\lambda_{n}=\mu_{f}(n) g_{\xi}(n)$, where

$$
g_{\xi}(n)= \begin{cases}1 & \text { if } 1 \leq n \leq \xi, \\ \frac{\log \left(\xi^{2} / n\right)}{\log \xi} & \text { if } \xi \leq n \leq \xi^{2}, \\ 0 & \text { if } n \geq \xi^{2},\end{cases}
$$

and $\xi=T^{\theta}, 0<\theta<1 / 4$ will be specified later.

We define the mollifier

$$
M_{\xi^{2}}(s)=\sum_{v} \frac{\lambda_{v}}{v^{s}}
$$

where $k / 2+A / \log \xi \leq \sigma \leq k / 2+\delta$, and $A, \delta^{-1}$ are sufficiently large positive numbers.

Using the multiplicativity of the Hecke eigenvalues $a_{n}$ [11] and the definition of $P(n, s)$,

$$
\begin{gathered}
P(n, s)=\prod_{p^{r} \| n} P\left(p^{r}, s\right) \\
P\left(p^{r}, s\right)=\left(\sum_{j=0}^{\infty} a_{p^{j+r}} a_{p^{j}} p^{-j s}\right)\left(\sum_{j=0}^{\infty} a_{p^{j}}^{2} p^{-j s}\right)^{-1},
\end{gathered}
$$

we easily have

$$
P(p, s)=\frac{a_{p}}{1+p^{k-1-s}}, \quad P\left(p^{2}, s\right)=\frac{a_{p}^{2}}{1+p^{k-1-s}}-p^{k-1} .
$$

We have, using Lemma 2.1,

$$
\begin{aligned}
& \int_{-\infty}^{\infty} \psi_{U}\left(\frac{t}{T}\right)\left|L_{f}(\sigma+i t)\right|^{2}\left|M_{\xi^{2}}(\sigma+i t)\right|^{2} d t \\
= & \sum_{v_{1}, v_{2} \leq \xi^{2}} \frac{\lambda_{v_{1}} \lambda_{v_{2}}}{\left(v_{1} v_{2}\right)^{2 \sigma}}\left(v_{1}, v_{2}\right)^{2 \sigma} D_{v_{1} /\left(v_{1}, v_{2}\right), v_{2} /\left(v_{1}, v_{2}\right)}(2 \sigma) \int_{-\infty}^{\infty} \psi_{U}\left(\frac{t}{T}\right) d t \\
& +\sum_{v_{1}, v_{2} \leq \xi^{2}} \frac{\lambda_{v_{1}} \lambda_{v_{2}}}{\left(v_{1} v_{2}\right)^{k}}\left(v_{1}, v_{2}\right)^{2(k-\sigma)} D_{v_{1} /\left(v_{1}, v_{2}\right), v_{2} /\left(v_{1}, v_{2}\right)}(2 k-2 \sigma) \\
& \times \int_{-\infty}^{\infty} \psi_{U}\left(\frac{t}{T}\right)\left(\frac{t}{2 \pi}\right)^{2 k-4 \sigma} d t+O\left(T^{4 / 5} U^{3} \xi^{14} \log ^{3} \xi\right) \\
= & S_{1}+S_{2}+S_{3},
\end{aligned}
$$

say. Since we have

$$
D_{v_{1} /\left(v_{1}, v_{2}\right), v_{2} /\left(v_{1}, v_{2}\right)}(2 \sigma)=D(2 \sigma) P\left(\frac{v_{1}}{\left(v_{1}, v_{2}\right)}, 2 \sigma\right) P\left(\frac{v_{2}}{\left(v_{1}, v_{2}\right)}, 2 \sigma\right),
$$


where

$$
D(s)=D_{11}(s)=\sum_{l=1}^{\infty} \frac{a_{l}^{2}}{l^{s}},
$$

it follows by Möbius inversion that

$$
\begin{aligned}
S_{1}= & D(2 \sigma) \int_{-\infty}^{\infty} \psi_{U}\left(\frac{t}{T}\right) d t \\
& \times \sum_{v_{1}, v_{2} \leq \xi^{2}} \frac{\lambda_{v_{1}} \lambda_{v_{2}}}{\left(v_{1} v_{2}\right)^{2 \sigma}}\left(v_{1}, v_{2}\right)^{2 \sigma} P\left(\frac{v_{1}}{\left(v_{1}, v_{2}\right)}, 2 \sigma\right) P\left(\frac{v_{2}}{\left(v_{1}, v_{2}\right)}, 2 \sigma\right) \\
= & D(2 \sigma) \int_{-\infty}^{\infty} \psi_{U}\left(\frac{t}{T}\right) d t \\
& \times \sum_{v_{1}, v_{2} \leq \xi^{2}} \frac{\lambda_{v_{1}} \lambda_{v_{2}}}{\left(v_{1} v_{2}\right)^{2 \sigma}} \sum_{r\left|v_{1}, r\right| v_{2}} \sum_{l \mid r} \mu(l)\left(\frac{r}{l}\right)^{2 \sigma} P\left(\frac{v_{1}}{r / l}, 2 \sigma\right) P\left(\frac{v_{2}}{r / l}, 2 \sigma\right) \\
= & D(2 \sigma) \int_{-\infty}^{\infty} \psi_{U}\left(\frac{t}{T}\right) d t \\
& \times \sum_{r \leq \xi^{2}, r \text { cubefree } l \mid r} \sum_{l} \mu(l)\left(\frac{r}{l}\right)^{2 \sigma}\left(\sum_{r \mid v} \frac{\lambda_{v}}{v^{2 \sigma}} P\left(\frac{v}{r / l}, 2 \sigma\right)\right)^{2} .
\end{aligned}
$$

Similarly

$$
\begin{aligned}
S_{2}= & D(2 k-2 \sigma) \int_{-\infty}^{\infty} \psi_{U}\left(\frac{t}{T}\right)\left(\frac{t}{2 \pi}\right)^{2 k-4 \sigma} d t \\
& \times \sum_{r \leq \xi^{2}, r \text { cubefree } l \mid r} \sum_{l} \mu(l)\left(\frac{r}{l}\right)^{2(k-\sigma)}\left(\sum_{r \mid v} \frac{\lambda_{v}}{v^{k}} P\left(\frac{v}{r / l}, 2(k-\sigma)\right)\right)^{2} .
\end{aligned}
$$

We distinguish two cases: (a) $r \leq \xi$, and (b) $\xi<r \leq \xi^{2}$.

First consider the case (a) $r \leq \xi$. We deduce that, since

$$
\frac{1}{2 \pi i} \int_{(2)} \frac{y^{s}}{s^{2}} d s= \begin{cases}\log y, & y \geq 1, \\ 0, & 0<y \leq 1,\end{cases}
$$

we have

$$
\begin{aligned}
& \sum_{v} \frac{\lambda_{r v}}{(r v)^{2 \sigma}} P(l v, 2 \sigma) \\
& \quad=\sum_{v} \frac{\mu_{f}(r v) g_{\xi}(r v)}{(r v)^{2 \sigma}} P(l v, 2 \sigma)
\end{aligned}
$$




$$
\begin{aligned}
= & \frac{1}{2 \pi i} \int_{(2)} \frac{\xi^{s}\left(\xi^{s}-1\right)}{s^{2}}\left(\sum_{v} \frac{\mu_{f}(r v)}{(r v)^{2 \sigma+s}} P(l v, 2 \sigma)\right) \frac{d s}{\log \xi} \\
= & \sum_{v} \frac{\mu_{f}(r v)}{(r v)^{2 \sigma}} P(l v, 2 \sigma) \\
& +\frac{1}{2 \pi i} \int_{\Gamma} \frac{(\xi / r)^{s}\left(\xi^{s}-1\right)}{s^{2}}\left(\sum_{v} \frac{\mu_{f}(r v)}{(r v)^{2 \sigma}} \cdot \frac{1}{v^{s}} P(l v, 2 \sigma)\right) \frac{d s}{\log \xi},
\end{aligned}
$$

where $\Gamma$ denotes the path $\{i x,|x| \geq \delta\} \cup\left\{\delta e^{i \theta}, \pi / 2 \leq \theta \leq 3 \pi / 2\right\}$, and $\delta$ is sufficiently small.

We observe that $\left(p^{e_{p}(r)}\left\|r, p^{e_{p}(l)}\right\| l\right)$

$$
\begin{aligned}
\sum_{v} \frac{\mu_{f}(r v)}{(r v)^{2 \sigma}} P(l v, 2 \sigma) & \\
= & \prod_{p}\left(1+\frac{\mu_{f}(p)}{p^{2 \sigma}} P(p, 2 \sigma)+\frac{\mu_{f}\left(p^{2}\right)}{p^{4 \sigma}} P\left(p^{2}, 2 \sigma\right)\right) \\
& \times \prod_{p \mid r} \frac{\frac{\mu_{f}\left(p^{e_{p}(r)}\right)}{\left(p^{e_{p}(r)}\right)^{2 \sigma}} P\left(p^{e_{p}(l)}, 2 \sigma\right)+\frac{\mu_{f}\left(p^{e_{p}(r)+1}\right)}{\left(p^{e_{p}(r)+1}\right)^{2 \sigma}} P\left(p^{e_{p}(l)+1}, 2 \sigma\right)}{1+\frac{\mu_{f}(p)}{p^{2 \sigma}} P(p, 2 \sigma)+\frac{\mu_{f}\left(p^{2}\right)}{p^{4 \sigma}} P\left(p^{2}, 2 \sigma\right)} \\
= & \frac{1}{D(2 \sigma)} u(r, l, 2 \sigma),
\end{aligned}
$$

say. Similarly

$$
\begin{aligned}
\sum_{v} \frac{\mu_{f}(r v)}{(r v)^{2 \sigma}} \cdot \frac{1}{v^{s}} P(l v, 2 \sigma) \\
=\prod_{p}\left(1+\frac{\mu_{f}(p)}{p^{2 \sigma+s}} P(p, 2 \sigma)+\frac{\mu_{f}\left(p^{2}\right)}{p^{4 \sigma+2 s}} P\left(p^{2}, 2 \sigma\right)\right) \\
\quad \times \prod_{p \mid r} \frac{\frac{\mu_{f}\left(p^{e_{p}(r)}\right)}{\left(p^{e_{p}(r)}\right)^{2 \sigma}} P\left(p^{e_{p}(l)}, 2 \sigma\right)+\frac{\mu_{f}\left(p^{e_{p}(r)+1}\right)}{\left(p^{e_{p}(r)+1}\right)^{2 \sigma} p^{s}} P\left(p^{e_{p}(l)+1}, 2 \sigma\right)}{1+\frac{\mu_{f}(p)}{p^{2 \sigma+s}} P(p, 2 \sigma)+\frac{\mu_{f}\left(p^{2}\right)}{p^{4 \sigma+2 s}} P\left(p^{2}, 2 \sigma\right)} \\
=G(s) v(r, l, 2 \sigma, s),
\end{aligned}
$$

say.

It is easily verified that, for $\Re s>-1 / 2$,

$$
G(s)=\frac{1}{D(2 \sigma+s)} \prod_{p}\left(1+O\left(\frac{1}{p^{2(1+\Re s)}}\right)\right) .
$$


We have, by Cauchy's inequality,

$$
\begin{aligned}
\left(\sum_{v} \frac{\lambda_{r v}}{(r v)^{2 \sigma}} P(l v, 2 \sigma)\right)^{2} \ll & \left|\sum_{v} \frac{\mu_{f}(r v)}{(r v)^{2 \sigma}} P(l v, 2 \sigma)\right|^{2}+\int_{\Gamma}\left|\left(\frac{\xi}{r}\right)^{s} \frac{\xi^{s}-1}{s^{2}}\right| \\
\times & \left|\sum_{v} \frac{\mu_{f}(r v)}{(r v)^{2 \sigma} v^{s}} P(l v, 2 \sigma)\right|^{2} \frac{|d s|}{\log ^{2} \xi} .
\end{aligned}
$$

For $r$ cubefree, $r=r_{1} r_{2}^{2}, \mu\left(r_{1} r_{2}\right) \neq 0$, we infer that

$$
\begin{aligned}
& \sum_{l \mid r}|\mu(l)|\left(\frac{r}{l}\right)^{2 \sigma}\left|\sum_{v} \frac{\mu_{f}(r v)}{(r v)^{2 \sigma}} P(l v, 2 \sigma)\right|^{2} \\
& \ll \prod_{p \mid r}\left(1+\frac{1}{p^{3 / 4}}\right) \frac{1}{D(2 \sigma)^{2}}\left(\sum_{t \mid r_{1}} \frac{a_{t}^{2}}{t^{2 \sigma}}\left(\frac{r_{1}}{t}\right)^{-3}\right) r_{2}^{2(k-1)-4 \sigma}, \\
& \sum_{l \mid r}|\mu(l)|\left(\frac{r}{l}\right)^{2 \sigma}\left|\sum_{v} \frac{\mu_{f}(r v)}{(r v)^{2 \sigma} v^{s}} P(l v, 2 \sigma)\right|^{2} \\
& \ll \prod_{p \mid r}\left(1+\frac{1}{p^{3 / 4}}\right) \frac{1}{|D(2 \sigma+s)|^{2}}\left(\sum_{t \mid r_{1}} \frac{a_{t}^{2}}{t^{2 \sigma}}\left(\frac{r_{1}}{t}\right)^{-3}\right) r_{2}^{2(k-1)-4 \sigma} .
\end{aligned}
$$

From the zero-free region result for $D(s)$ (see, for example, [17], Theorem 5.1) and a standard argument (due to Landau, see [21], §3.9 and §3.11), we have

$$
D(s) \neq 0, \quad 1 / D(s) \ll \log (|y|+3),
$$

for $s=x+i y, x \geq k-2 \delta / \log (|y|+3)$. Note that

$$
\begin{aligned}
\sum_{r \leq \xi} \frac{a_{r}^{2}}{r^{2 \sigma}} \prod_{p \mid r}\left(1+\frac{1}{p^{3 / 4}}\right) & =\sum_{r \leq \xi} \frac{a_{r}^{2}}{r^{2 \sigma}} \sum_{u \mid r} \frac{|\mu(u)|}{u^{3 / 4}}=\sum_{u \leq \xi} \frac{|\mu(u)|}{u^{3 / 4+2 \sigma}} \sum_{r \leq \xi / u} \frac{a_{r u}^{2}}{r^{2 \sigma}} \\
& \ll \sum_{u} \frac{1}{u^{3 / 4+2 \sigma}}\left(\sum_{(r, u)=1} \frac{a_{r}^{2}}{r^{2 \sigma}}\right)\left(\sum_{r \mid u^{\infty}} \frac{a_{r u}^{2}}{r^{2 \sigma}}\right) \\
& \ll D(2 \sigma) \sum_{u} \frac{d^{2}(u)}{u^{3 / 4+2 \sigma}} \sum_{r \mid u^{\infty}} \frac{(r u)^{k-1} d^{2}(r)}{r^{2 \sigma}} \\
& \ll D(2 \sigma) \sum_{u} \frac{d^{2}(u)}{u^{7 / 4}} \sum_{r \mid u^{\infty}} \frac{d^{2}(r)}{r} \ll D(2 \sigma) .
\end{aligned}
$$

Here we have used Deligne's bound for the Hecke eigenvalues $a_{r u}$, but it is clear that the weaker and more elementary bound $a_{r} \ll r^{(k-1) / 2+1 / 4+\varepsilon}$ suffices for the same purpose. Hence 
$\sum_{r_{1} r_{2}^{2} \leq \xi, \mu\left(r_{1} r_{2}\right) \neq 0} d\left(r_{2}\right) r_{2}^{2(k-1)-4 \sigma} \sum_{t \mid r_{1}} \frac{a_{t}^{2}}{t^{2 \sigma}} \prod_{p \mid t}\left(1+\frac{1}{p^{3 / 4}}\right)\left(\frac{r_{1}}{t}\right)^{-3} \prod_{p \mid \frac{r_{1}}{t}}\left(1+\frac{1}{p^{3 / 4}}\right)$
$\ll \sum_{t \leq \xi} \frac{a_{t}^{2}}{t^{2 \sigma}} \prod_{p \mid t}\left(1+\frac{1}{p^{3 / 4}}\right) \sum_{r \leq \xi / t} \frac{d(r)}{r^{3}} \sum_{r_{2} \leq \sqrt{\xi}} \frac{d\left(r_{2}\right)}{r_{2}^{2}} \ll D(2 \sigma)$.

Thus,

$$
D(2 \sigma) \sum_{r \leq \xi} \sum_{l \mid r}|\mu(l)|\left(\frac{r}{l}\right)^{2 \sigma}\left|\sum_{v} \frac{\lambda_{r v}}{(r v)^{2 \sigma}} P(l v, 2 \sigma)\right|^{2} \ll 1 .
$$

(Note that $2 \sigma-k \gg 1 / \log \xi$.)

In case (b), $\xi<r \leq \xi^{2}$, we have

$$
\sum_{v} \frac{\lambda_{r v}}{(r v)^{2 \sigma}} P(l v, 2 \sigma)=\frac{1}{2 \pi i} \int_{(2)}\left(\frac{\xi^{2}}{r}\right)^{s} \frac{1}{s^{2}}\left(\sum_{v} \frac{\mu_{f}(r v)}{(r v)^{2 \sigma} v^{s}} P(l v, 2 \sigma)\right) \frac{d s}{\log \xi} .
$$

The treatment is the same except that the above integrand has a double pole at $s=0$. Using $\sum_{p \mid r} \log p / p \ll \log \log r$, we can establish that

$$
D(2 \sigma) \sum_{\xi<r \leq \xi^{2}} \sum_{l \mid r}|\mu(l)|\left(\frac{r}{l}\right)^{2 \sigma}\left|\sum_{v} \frac{\lambda_{r v}}{(r v)^{2 \sigma}} P(l v, 2 \sigma)\right|^{2} \ll 1 .
$$

Hence $S_{1} \ll T$. Similarly, $S_{2} \ll T$. If we choose $\psi_{U}(t / T)$ to be the majorant of the characteristic function of $[T, 2 T]$ (here $U \ll 1$ ), then we have, with $\xi=T^{1 / 72}$

$$
\int_{T}^{2 T}\left|L_{f}(\sigma+i t)\right|^{2}\left|M_{\xi^{2}}(\sigma+i t)\right|^{2} d t \ll T .
$$

In particular, we have

Lemma 3.1. Let $\sigma=k / 2+A / \log T$. Then

$$
\int_{T}^{2 T}\left|L_{f}(\sigma+i t) M_{\xi^{2}}(\sigma+i t)-1\right|^{2} d t \ll T .
$$

We also have

LEMMA 3.2.

$$
\int_{T}^{2 T}\left|L_{f}(k / 2+1+i t) M_{\xi^{2}}(k / 2+1+i t)-1\right|^{2} d t \ll T^{1-1 / 72} .
$$

Pr o of. Lemma 3.2 follows immediately from the equality (see [16])

$$
\int_{0}^{T}\left|\sum_{n=1}^{\infty} a_{n} n^{i t}\right|^{2} d t=\sum_{n=1}^{\infty}\left|a_{n}\right|^{2}(T+O(n)) .
$$


From Lemma 3.1, Lemma 3.2 and an easy modification of the classical convexity theorem (see $[21], \S 7.8$ ), we deduce that

Theorem 3.3. We have

$$
\int_{T}^{2 T}\left|L_{f}(\sigma+i t) M_{\xi^{2}}(\sigma+i t)-1\right|^{2} d t \ll T^{1-\frac{1}{72}(\sigma-k / 2)},
$$

uniformly for $k / 2+A / \log T \leq \sigma \leq k / 2+1$.

Now we are in a position to prove our main theorem.

TheOREM 3.4. We have

$$
N_{f}(\sigma, T) \ll T^{1-\frac{1}{72}(\sigma-k / 2)} \log T,
$$

uniformly for $k / 2 \leq \sigma \leq(k+1) / 2$.

First, we show the following proposition.

Proposition 3.5. We have

$$
\int_{\sigma}^{(k+1) / 2} N_{f}\left(\sigma^{\prime}, T\right) d \sigma^{\prime} \ll T^{1-\frac{1}{72}(\sigma-k / 2)},
$$

uniformly for $k / 2 \leq \sigma \leq(k+1) / 2$.

P r o of. It suffices to prove that

$$
\int_{\sigma}^{(k+1) / 2}\left(N_{f}\left(\sigma^{\prime}, 2 T\right)-N_{f}\left(\sigma^{\prime}, T\right)\right) d \sigma^{\prime} \ll T^{1-\frac{1}{72}(\sigma-k / 2)},
$$

for $k / 2+A / \log \xi \leq \sigma \leq(k+1) / 2$.

Let $\Phi(s)=1-\left(L_{f}(s) M_{\xi^{2}}(s)-1\right)^{2}$. The zeros of $L_{f}(s)$ occur among those of $\Phi(s)$, with at least the same multiplicities. By Littlewood's lemma concerning the number of zeros of an analytic function in a rectangle [22], we have

$$
\begin{aligned}
\int_{\sigma}^{(k+1) / 2}\left(N_{f}\left(\sigma^{\prime}, 2 T\right)\right. & \left.-N_{f}\left(\sigma^{\prime}, T\right)\right) d \sigma^{\prime} \\
\leq & \frac{1}{2 \pi} \int_{T}^{2 T} \log |\Phi(\sigma+i t)| d t+\frac{1}{2 \pi} \int_{\sigma}^{\infty} \arg \Phi\left(\sigma^{\prime}+2 i T\right) d \sigma^{\prime} \\
& -\frac{1}{2 \pi} \int_{\sigma}^{\infty} \arg \Phi\left(\sigma^{\prime}+i T\right) d \sigma^{\prime} .
\end{aligned}
$$

In the range $((k+1) / 2+4, \infty)$, we see that

$$
\arg \Phi\left(\sigma^{\prime}+i t\right)=O\left(2^{-\sigma^{\prime}}\right) .
$$


Hence this part of the integral is $O(1)$. In the range $(k / 2,(k+1) / 2+4)$, it follows from Jensen's theorem [22] and a standard argument (see [19]) that

$$
\arg \Phi\left(\sigma^{\prime}+i T\right)=O(\log T) .
$$

We deduce that

$$
\int_{\sigma}^{\infty} \arg \Phi\left(\sigma^{\prime}+i T\right) d \sigma^{\prime} \ll \log T .
$$

Finally, since $\log (1+|x|) \leq|x|$,

$$
\begin{aligned}
\int_{T}^{2 T} \log |\Phi(\sigma+i t)| d t & \leq \int_{T}^{2 T}\left|L_{f}(\sigma+i t) M_{\xi^{2}}(\sigma+i t)-1\right|^{2} d t \\
& =O\left(T^{1-\frac{1}{72}(\sigma-k / 2)}\right) .
\end{aligned}
$$

This proves the proposition.

Proof of Theorem 3.4. It suffices to assume that $\sigma-k / 2 \geq 1 / \log T$. Thus,

$$
\begin{aligned}
N_{f}(\sigma, T) & \leq \log T \int_{\sigma-1 / \log T}^{\sigma} N_{f}\left(\sigma^{\prime}, T\right) d \sigma^{\prime} \\
& \leq \log T \int_{\sigma-1 / \log T}^{(k+1) / 2} N_{f}\left(\sigma^{\prime}, T\right) d \sigma^{\prime} \\
& \ll T^{1-\frac{1}{72}(\sigma-k / 2)} \log T .
\end{aligned}
$$

Our proof is now complete.

4. Appendix. In this section, we will give a simple proof of Hafner's result which is used in Section 2 without appealing to the spectral theory of the Laplacian acting on $L^{2}\left(\boldsymbol{\Gamma}_{0}(a, b) \backslash \mathbf{H}\right)$. Our approach is based upon the delta-symbol method introduced by Duke-Friedlander-Iwaniec [5] and does not require a discussion of exceptional eigenvalues for the congruence subgroups. Instead we only need Weil's bound for the Kloosterman sums. Our result is quantitatively a little weaker than Hafner's but is sufficient for our application. Furthermore, our method can as well be applied to the case when $a_{n}$ is the Fourier coefficient of a Maass form so it appears to be of independent interest.

Let $a_{n}$ be the $n$th Fourier coefficient of a (holomorphic) Hecke eigenform of weight $k$. We consider the sum

$$
\sum_{m \mu-n \nu=l, x \leq n \leq 2 x} a_{n} a_{m}, \quad \mu, \nu, l>0, x \geq 10 .
$$


Let $g(\xi)$ be a smooth function on $\mathbb{R}^{1}$ with compact support such that $0 \leq g(\xi) \leq 1 ; g(\xi)=1$ if $x \leq \xi \leq 2 x ; \operatorname{supp}(g(\xi)) \subset\left[x-x^{1-\theta}, 2 x+x^{1-\theta}\right]$ for some $0<\theta<1$; and $g^{(p)}(\xi) \ll_{p}\left(x^{1-\theta}\right)^{-p}, p \geq 0$. Then we have

$$
\begin{aligned}
& \sum_{m \mu-n \nu=l, x \leq n \leq 2 x} a_{n} a_{m} \\
& =\sum_{m \mu-n \nu=l} a_{n} a_{m} g(n)+O\left(\left(\frac{x \nu+l}{\mu}\right)^{(k-1) / 2+\varepsilon} x^{(k-1) / 2+\varepsilon} x^{1-\theta}\right) .
\end{aligned}
$$

Let $h(\xi)$ be another smooth function with compact support such that $0 \leq h(\xi) \leq 1 ; h(\xi)=1$, if $\left(\frac{3}{4} x \nu+l\right) / \mu \leq \xi \leq\left(\frac{9}{4} x \nu+l\right) / \mu ; \operatorname{supp}(h(\xi)) \subset$ $\left[\left(\frac{1}{2} x \nu+l\right) / \mu,\left(\frac{5}{2} x \mu+l\right) / \nu\right]$; and $h^{(p)}(\xi) \ll_{p}(x \nu / \mu)^{-p}, p \geq 0$. Clearly we have

$$
\sum_{m \mu-n \nu=l} a_{n} a_{m} g(n)=\sum_{m \mu-n \nu=l} a_{n} a_{m} g(n) h(m) .
$$

Next we will recall the delta-symbol method introduced in [5].

Define

$$
\delta(n)= \begin{cases}1 & \text { if } n=0 \\ 0 & \text { if } n \neq 0 .\end{cases}
$$

Let $\omega(t)$ be an even function on $\mathbb{R}^{1}$ with $\omega(0)=0$ and compactly supported such that $\sum_{k=1}^{\infty} \omega(k)=1$. Let

$$
\delta_{k}(n)=\omega(k)-\omega\left(\frac{n}{k}\right) .
$$

Then clearly $\delta(n)=\sum_{k \mid n} \delta_{k}(n)$. Thus

$$
\delta(n)=\sum_{k} k^{-1} \sum_{h \bmod k} e\left(\frac{h n}{k}\right) \delta_{k}(n) .
$$

Put

$$
\Delta_{c}(n)=\sum_{r} r^{-1} \delta_{c r}(n)
$$

Writing $r=(h, k), a=h / r, c=k / r$, we have

$$
\delta(n)=\sum_{c} c^{-1} \sum_{a \bmod c}^{*} e\left(\frac{a n}{c}\right) \Delta_{c}(n) .
$$

We will apply the above identity to integers $|n|<N / 2$, say, with $\omega(t)$ supported on $K / 2<|t|<K$, and whose derivatives satisfy $\omega^{(j)}(t) \ll K^{-j-1}$. Now, $\delta_{k}(n)$ vanishes except for $1 \leq k<\max (K, N / K)=K$ by choosing $K=N^{1 / 2}$. Hence $\Delta_{c}(n)$ vanishes except for $1 \leq c<K$ and $\Delta_{c}(n) \ll$ $K^{-1} \log K$. Let $\Delta_{1}=x \nu / \mu, \Delta_{2}=x^{1-\theta}, \Delta=\min \left(\Delta_{1}, \Delta_{2}\right)$. We infer that, 
by (10) and (11),

$$
\begin{aligned}
S & =\sum_{m \mu-n \nu=l} a_{n} a_{m} g(n) h(m) \\
& =\sum_{m, n} a_{n} a_{m} g(n) h(m) \delta(m \mu-n \nu-l)=\sum_{c} c^{-1} S_{c},
\end{aligned}
$$

where

$$
\begin{aligned}
S_{c} & =\sum_{a \bmod c}^{*} \sum_{m, n} a_{m} a_{n} g(n) h(m) e\left(\frac{a(m \mu-n \nu-l)}{c}\right) \Delta_{c}(m \mu-n \nu-l) \\
& =\sum_{a \bmod c}^{*} e\left(\frac{-a l}{c}\right) \sum_{m, n} b_{m} b_{n} e\left(\frac{a}{c}(m \mu-n \nu)\right) F(m, n),
\end{aligned}
$$

with $b_{m}=a_{m} m^{-(k-1) / 2}$ and

$$
F(m, n)=(m n)^{(k-1) / 2} h(m) g(n) \Delta_{c}(\mu m-\nu n-l) .
$$

Define $\gamma=\max (\mu, \nu), K^{2}=N=8 x \gamma l$. It is easy to see that

$$
\frac{d}{d n} \Delta_{c}(n) \begin{cases}\ll 1 / K|n| & \text { if }|n| \gg K c, \\ =0 & \text { otherwise. }\end{cases}
$$

We have, for $i+j \geq 1$,

$$
\begin{aligned}
& \frac{\partial^{i+j}}{\partial \xi^{i} \partial \eta^{j}} F(\xi, \eta) \\
& \ll\left(\frac{x \nu+l}{\mu}\right)^{(k-1) / 2} x^{(k-1) / 2} K^{-1}\left(\frac{\Delta c}{K}\right)^{-i-j+1}\left(\frac{\mu+\nu}{|\mu \xi-\nu \eta-l|}+\frac{1}{\Delta}\right)
\end{aligned}
$$

if $|\mu \xi-\nu \eta-l| \gg K c$, and without the term $(\mu+\nu) /|\mu \xi-\nu \eta-l|$ if otherwise. We need the following Poisson-type formula [4]:

LEMMA 4.1. Let $F$ be a smooth and compactly supported function on $\mathbb{R}^{+}$. For any integers $c \geq 1$ and $(a, c)=1$ we have

$$
\sum_{m} b_{m} e\left(\frac{a m}{c}\right) F(m)=\sum_{r} b_{r} e\left(\frac{-\bar{a} r}{c}\right) \breve{F}(r),
$$

where $a \bar{a} \equiv 1(\bmod c)$ and $\breve{F}(r)$ is the Hankel-type transform

$$
\breve{F}(y)=2 \pi i^{k} c^{-1} \int_{0}^{\infty} F(x) J_{k-1}\left(\frac{4 \pi}{c} \sqrt{x y}\right) d x,
$$

where $J_{\nu}(z)$ is the usual Bessel function.

Applying Lemma 4.1 in each variable $m, n$ in (12), we deduce that

$$
S_{c}=\sum_{a \bmod c}^{*} e\left(\frac{a l}{c}\right) \sum_{r_{1}, r_{2}} b_{r_{1}} b_{r_{2}} e\left(\frac{\overline{a \mu}}{c_{1}} r_{1}-\frac{\overline{a \nu_{1}}}{c_{2}} r_{2}\right) \breve{F}\left(r_{1}, r_{2}\right),
$$


where

and

$$
\mu_{1}=\frac{\mu}{(\mu, c)}, \quad c_{1}=\frac{c}{(\mu, c)}, \quad \nu_{1}=\frac{\nu}{(\nu, c)}, \quad c_{2}=\frac{c}{(\nu, c)}
$$

(16) $\breve{F}\left(r_{1}, r_{2}\right)$

$$
=\frac{4 \pi^{2}}{c_{1} c_{2}} \int_{0}^{\infty} \int_{0}^{\infty} F\left(x_{1}, x_{2}\right) J_{k-1}\left(\frac{4 \pi}{c_{1}} \sqrt{x_{1} r_{1}}\right) J_{k-1}\left(\frac{4 \pi}{c_{2}} \sqrt{x_{2} r_{2}}\right) d x_{1} d x_{2} .
$$

By the recurrence formula

$$
\frac{d}{d z}\left(z^{\nu} J_{\nu}(z)\right)=z^{\nu} J_{\nu-1}(z)
$$

and the bound $J_{\nu}(z) \ll(1+z)^{-1 / 2}$, we obtain, by partial integration twice in each variable in (16) and using (14),

$$
\sum_{r_{1}, r_{2}} b_{r_{1}} b_{r_{2}}\left|\breve{F}\left(r_{1}, r_{2}\right)\right| \ll K\left(l \gamma x^{\theta+\varepsilon}\right)^{9 / 4}\left(\frac{x \nu+l}{\mu}\right)^{(k-1) / 2} x^{(k-1) / 2} .
$$

The sum over $a$ in (15) is a Kloosterman sum $S(l, *, c)$ to which we apply Weil's bound. Thus, we infer that

$$
S_{c} \ll(l, c)^{1 / 2} c^{1 / 2} \tau(c)(x l \gamma)^{1 / 2}\left(\gamma l x^{\theta+\varepsilon}\right)^{9 / 4}\left(\frac{x \nu+l}{\mu}\right)^{(k-1) / 2} x^{(k-1) / 2} .
$$

Hence

$$
\begin{aligned}
S & \ll(x l \gamma)^{3 / 4}\left(l \gamma x^{\theta+\varepsilon}\right)^{9 / 4}\left(\frac{x \nu+l}{\mu}\right)^{(k-1) / 2} x^{(k-1) / 2} \\
& \ll\left(\frac{x \nu+l}{\mu}\right)^{(k-1) / 2} x^{(k-1) / 2} x^{3 / 4+9 \theta / 4+\varepsilon} \gamma^{3} l^{3} .
\end{aligned}
$$

We conclude that

$$
\begin{aligned}
\sum_{n \leq x} a_{n} a_{(n \nu+l) / \mu} & \ll\left(\frac{x \nu+l}{\mu}\right)^{(k-1) / 2} x^{(k-1) / 2}\left(l^{3} x^{3 / 4+9 \theta / 4+\varepsilon} \gamma^{3}+x^{1-\theta+\varepsilon}\right) \\
& \ll\left(\frac{x \nu+l}{\mu}\right)^{(k-1) / 2} x^{(k-1) / 2} \gamma^{3} l^{3} x^{13 / 14},
\end{aligned}
$$

on taking $\theta=1 / 13$. Finally, since for $\Re s>k$,

we obtain

$$
\begin{aligned}
D_{\mu, \nu}(s, l):=\sum_{n=1}^{\infty} \frac{a_{n} a_{(n \nu+l) / \mu}}{(n \nu+l / 2)^{s}} & =\int_{1 / 2}^{\infty} \frac{1}{(x \nu+l / 2)^{s}} d\left(\sum_{n \leq x} a_{n} a_{(n \nu+l) / \mu}\right) \\
& =s \nu \int_{1 / 2}^{\infty} \frac{\sum_{n \leq x} a_{n} a_{(n \nu+l) / \mu}}{(x \nu+l / 2)^{s+1}} d x,
\end{aligned}
$$


TheOREm 4.2. $D_{\mu, \nu}(s, l)$ can be analytically continued to $\Re s>k-1 / 14$, and for $\Re s>k-1 / 14, s=\sigma+i t$, we have

$$
D_{\mu, \nu}(s, l) \ll \frac{l^{3}|s|}{(\mu \nu)^{(k-1) / 2}}(\max (\mu, \nu))^{3} \frac{1}{\sigma-(k-1 / 14)} .
$$

\section{References}

[1] E. Bombieri and D. A. Hejhal, On the distribution of zeros of linear combinations of Euler products, preprint, 1993.

[2] P. Deligne, Formes modulaires et représentations l-adiques, Sém. Bourbaki, 1968/69, exposés 355 .

[3] —, La conjecture de Weil, I, Inst. Hautes Études Sci. Publ. Math. 43 (1974), 273-307.

[4] W. D u ke and H. Iw aniec, Bilinear forms in the Fourier coefficients of half-integral weight cusp forms and sums over primes, Math. Ann. 286 (1990), 783-802.

[5] W. Duke, J. Friedlander and H. Iwaniec, Bound for automorphic L-functions, Invent. Math. 112 (1993), 1-8.

[6] D. Farmer, Mean value of Dirichlet series associated with holomorphic cusp forms, J. Number Theory, to appear.

[7] A. Good, Approximative Funktionalgleichungen und Mittelwertsätze für Dirichletreihen, die Spitzenformen assoziiert sind, Comment. Math. Helv. 50 (1975), $327-361$.

[8] -, Beiträge zur Theorie der Dirichletreihen, die Spitzenformen zugeordnet sind, J. Number Theory 13 (1981), 18-65.

[9] G. H. Hardy, Note on Ramanujan's function $\tau(n)$, Proc. Cambridge Philos. Soc. 23 (1927), 675-680.

[10] E. Hecke, Über die Bestimmung Dirichletscher Reihen durch ihre Funktionalgleichung, Math. Ann. 112 (1936), 664-699.

[11] —, Über Modulfunktionen und die Dirichletschen Reihen mit Eulerscher Produktentwicklung, ibid. 114 (1937), 1-28.

[12] J. L. Hafner, Explicit estimates in the arithmetic theory of cusp forms and Poincaré series, ibid. 264 (1983), 9-20.

[13] - On the zeros (à la Selberg) of Dirichlet series attached to certain cusp forms, in: Topics in Analytic Number Theory, Austin, 1985, 127-164.

[14] M. Jutila, Zeros of the zeta-function near the critical line, in: Studies in Pure Mathematics to the Memory of Paul Turán, Birkhäuser, Basel, 1982, 385-394.

[15] C. G. Lekkerkerker, On the zeros of a class of Dirichlet series, Dissertation, Utrecht, 1955.

[16] H. L. Montgomery and R. C. Vaughan, Hilbert's inequality, J. London Math. Soc. 8 (1974), 73-82.

[17] C. J. Moreno, Explicit formulas in the theory of automorphic forms, in: Lecture Notes in Math. 626, Springer, 1977, 73-216.

[18] R. A. Rankin, Contributions to the theory of Ramanujan's function $\tau(n)$ and similar arithmetic functions, Proc. Cambridge Philos. Soc. 35 (1939), 357-372.

[19] A. Selberg, On the zeros of Riemann's zeta-function, in: Collected Papers, Vol. I, Springer, 1989, 85-141. 
[20] A. Selberg, Contributions to the theory of the Riemann zeta-function, in: Collected Papers, Vol. I, Springer, 1989, 214-280.

[21] E. C. Titchmarsh, The Theory of the Riemann Zeta-Function, Oxford, 1951.

[22] —, The Theory of Functions, Oxford, 1952.

MATHEMATICAL SCIENCES RESEARCH INSTITUTE

1000 CENTENNIAL DRIVE

BERKELEY, CALIFORNIA 94720

U.S.A.

Received on 6.6.1994

and in revised form on 28.10 .1994 